\title{
Research on Image Processing Algorithm Based on DSP
}

\section{Xinfeng Yang ${ }^{a}$ and Fengzhi Huang ${ }^{b}$}

\author{
School of Computer and Information Engineering, Nanyang Institute of Technology, Nanyang, \\ China \\ a1832370485@qq.com, b851663118@qq.com
}

Keywords: Digital image process; Filter; Enhancement; Segmentation

\begin{abstract}
This thesis mainly discusses the fundamental theories and key technologies of digital image processing algorithms, and discusses the methods of image filtering, image enhancement and image segmentation. To solve the large quantity of data and the complicated requirements in image processing algorithms, this paper takes TI's TMS320C6713 as the center processing unit to design a real-time image processing system. It can achieve the methods including median filtering, histogram equalization, edge detection, foreground extraction, and binarization. In CCS3.3 simulation environment, the results show that the algorithms designed achieve the desired functional requirements.
\end{abstract}

\section{Introduction}

Visual information accounts for a large proportion of the information that people get from the objective world. In the digital image processing system, the image processing speed has become the main factor affecting real-time [1]. In this paper, we study the basic theory and key techniques of common algorithms based on DSP digital image processing, image preprocessing and image segmentation of image sequences, and focus on the extraction of foreground images based on background templates and the methods and key technologies of image segmentation Sobel edge Detection and binarization.

\section{Hardware and Software System Architecture}

The software system architecture in this paper is the algorithm module used in image processing. The system will be 8-bit gray image directly into the DSP chip for image preprocessing and image processing. The software system workflow is as follows:

Through the system initialization, the parameters of the system set, the image input to the TMS320C6713 chip.

Preprocessing the input digital image, including filtering denoising, image histization and contrast enhancement.

Edge detection of the pre-processed image, using the background difference to extract the target image and its binarization.

Image preprocessing and image processing also include several algorithms, including preprocessing, gray histogram calculation and histogram equalization [2]. The purpose of image filtering is to suppress the noise of the target image under the condition of preserving the detail feature of the image [3]; The grayscale histogram is a function of the grayscale value, which describes the frequency of the gray value in the image; Histogram equalization is the non-linear stretching of the image, re-allocation of image pixel values, so that a certain gray-scale range of pixels are roughly the same [4].

Image processing includes edge detection, image difference and binarization of the three algorithms [5]. The purpose of edge detection is to identify the digital image in the brightness of the obvious point, highlight the edge of the image; Image difference is the use of background difference method to obtain the foreground image, more suitable for the background fixed occasions; Binarization is to achieve the normalization of pixels, reducing the amount of image information processing. 
Hardware system mainly by the processor, clock circuit, reset circuit, power circuit, watchdog and other components.

\section{Image Preprocessing Algorithm Design}

The main purpose of image preprocessing is to eliminate irrelevant information in the image, to restore useful information, to enhance the detectability of the information and to maximize the data. In this paper, image preprocessing is mainly for noise filtering, gray histogram calculation and histogram equalization [6-8].

\section{Median Filter}

Median filtering is a non-linear smoothing technique. The basic principle is to replace the value of the point of the digital image with the median value of each point in the neighborhood of the point, so that the surrounding pixel value is close to the value, thus eliminating the isolated noise point [9].

The algorithm implementation process is as follows:

First determine the shape and size of a filter window;

Sort the gray values $(\mathrm{x} 1, \mathrm{x} 2, \mathrm{x} 3, \ldots, \mathrm{xn})$ of the pixels in the window to find the median $\mathrm{s}=$ medin $\{\mathrm{x} 1, \mathrm{x} 2, \mathrm{x} 3, \ldots, \mathrm{xn}\}$;

All the pixels in the window gray value are set to $\mathrm{s}$.

The algorithm execution effect is shown in Fig. 1 and Fig. 2.

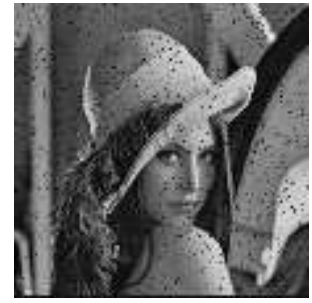

Figure 1. Original map before median filter

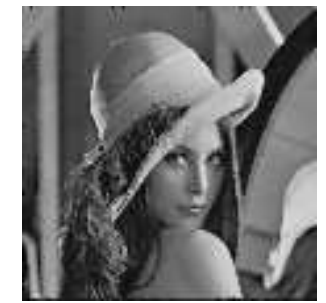

Figure 2. Effect map after median filter

\section{Histogram Statistics of Gray Image}

The histogram is used to represent the statistical plot of the gray value distribution of the image [10]. The abscissa of the histogram is the gray scale and the ordinate image type. For the continuous image, the ordinate is the probability density $P_{r}(r)$ of the gray value and the following relation holds:

$$
P_{r}(r)=\lim _{\Delta r \rightarrow 0} \frac{A(r+\Delta r)}{A \Delta r}, \int_{r_{\min }}^{r_{\max }} P_{r}(r) d r=1
$$

Wherein, $\mathrm{A}$ is the image area, and for the digital image, the ordinate is the number of pixels which gray value is $r_{r}$ or the probability $P_{r}\left(r_{r}\right)$ of occurrence of the gray value.

The algorithm implementation process is as follows:

Defines an array of 0-255, where each element corresponds to the value in the array and satisfies $\mathrm{t}[0]=0, \mathrm{t}[1]=1, \ldots, \mathrm{t}[255]=255$.

Find the number $N_{i}$ of pixels corresponding to the array elements $\mathrm{t}[0], \mathrm{t}[1], \ldots, \mathrm{t}[255]$.

Calculate the number of pixels $\mathrm{m}$ for the whole image.

Find the ratio $N_{i} / M$ of the pixels corresponding to each gray level to the total number of pixels, which is the histogram of the image. The effect of the algorithm is shown in Fig.3. 


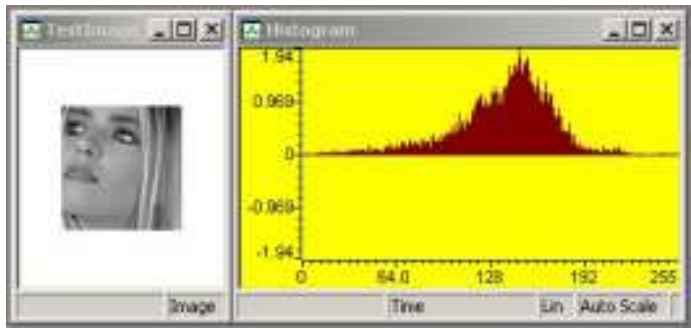

Figure 3. Histogram statistical effect map

\section{Histogram Equalization of Gray Image}

The grayscale histogram of the natural image is usually larger in the low-value gray-scale region, so that the details in the darker regions of the image are often unclear, and the gray scale with a smaller gray-scale frequency can be made larger The histogram tends to be consistent over the larger dynamic range to achieve the effect of enhancing the display of the image [11-12]. This method is called histogram equalization.

The algorithm flow is as follows:

The total number of pixels of the original image is $\mathrm{N}$, the number of gray levels is $\mathrm{L}$, and the frequency of the Kth gray scale is $N_{k}$.

If the gray level of the original image $f(x, y)$ at the pixel $(x, y)$ is $r_{k}$, the gray level of the image at the pixel $(x, y)$ after histogram equalization is: $S_{K}=T\left(r_{k}\right)=\sum_{j=1}^{k} \frac{N_{j}}{N} * 255$

The new value will be directly replaced by the original gray value, that is, to get the value of equalization.

Algorithm execution results as shown in Fig.4

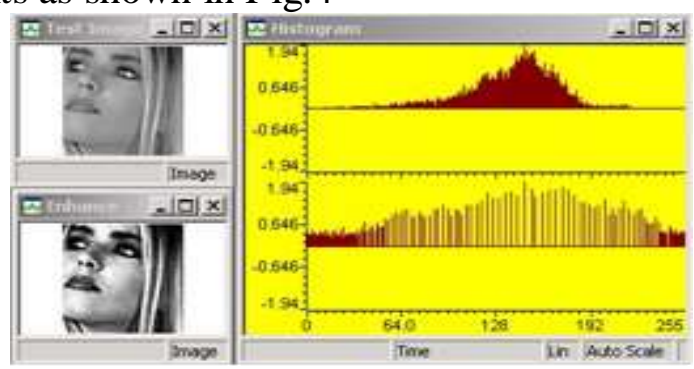

Figure 4. Histogram equalization effect map

\section{Image Processing}

Image processing has two purposes: one is to produce more suitable for people to observe and identify the image; the second is to be able to automatically identify and understand the image by the processor [13-14]. In order to extract more accurate image information, this paper uses image segmentation based on region and boundary.

\section{Edge Detection}

The edge of the image is the most basic feature of the image. Commonly used edge detection algorithms are generally based on the first order differential edge detection operator. Based on this article, the Sobel edge detection algorithm is adopted.

Algorithm implementation:

First, the method of determining the edge detection is to use different convolution cores in the $\mathrm{X}$ and Y directions, respectively, as follows:

$$
H_{X}:\left(\begin{array}{ccc}
-1 & -2 & -1 \\
0 & 0 & 0 \\
1 & 2 & 1
\end{array}\right) \quad H_{y}:\left(\begin{array}{lll}
-1 & 0 & 1 \\
-2 & 0 & 2 \\
-1 & 0 & 1
\end{array}\right)
$$

Wherein, $\mathrm{X}$ and $\mathrm{Y}$ respectively are the convolution cores in the $\mathrm{x}$ direction and the $\mathrm{y}$ direction. 
When a pixel is convoluted by $H_{x}$ and $H_{y}$ respectively, the values $f_{x}(x, y)$ and $f_{y}(x, y)$ in the horizontal and vertical directions are obtained, the new value of the pixel can be calculated using the following formula: $V=\sqrt{f_{x}(x, y)^{2}+f_{y}(x, y)^{2}}, d=\arctan \left(\frac{f_{x}(x, y)}{f_{y}(x, y)}\right)$.

Where $\mathrm{V}$ is the intensity of the pixel and $\mathrm{d}$ is the direction.

The resulting $\mathrm{V}$ value is the gray value of a pixel.

The algorithm effect is shown in Fig.5 and Fig.6:

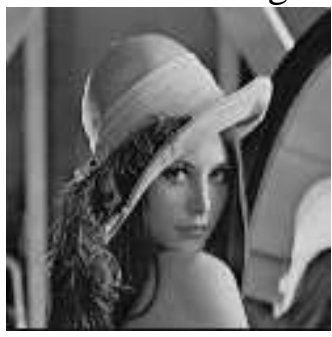

Figure 5. Original map

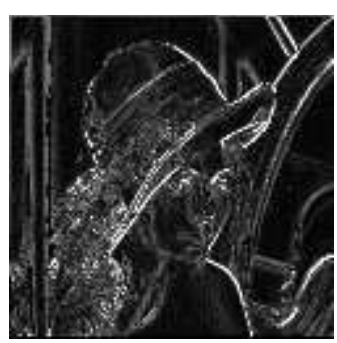

Figure 6. Effect map

\section{Extraction of Foreground Image Area Based on Background Template}

Algorithm implementation:

Read the background template and frame image. Set background image $B=\{b(x, y), x \in M, y \in N\}$; Set frame image $F=\{f(x, y), x \in M, y \in N\}$.

Set the difference image as $G, \quad G=\{g(x, y), x \in M, y \in N\}$, $g(x, y)=f(x, y)-b(x, y) \mid$, Wherein, $(x, y)$ is the coordinates of the image at any point, $f(x, y)$ is the gray value of the image $(x, y), g(x, y)$ is the gray value of the image $(x, y)$ after the difference.

The algorithm effect is shown in Fig.7- Fig.9.

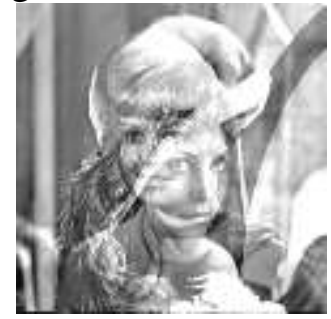

Figure 7. Original map

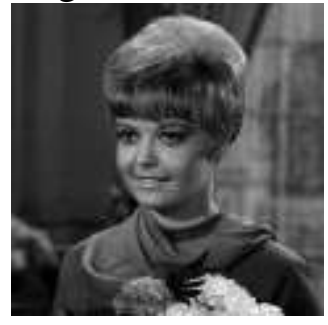

Figure 8. Template

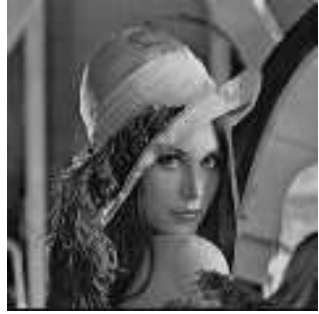

Figure 9. Effect map

\section{Extraction of Foreground Image Area Based on Background Template}

Algorithm implementation:

First find the highest gray level of the image L;

Then take each gray level from 0 to $\mathrm{L}$ as the threshold $\mathrm{t}$;

Calculate the respective pixel numbers $w_{0}$ and $u_{0}$ of the two classes separated by the threshold, and the mean values $W_{1}$ and $u_{1}$ in the entire gray scale image, and calculate the average gray scale of the entire image $u=w_{0} \times u_{0}+w_{1} \times u_{1}$;

Calculate the variance between two classes $b(t): b(t)=w_{0} \times\left(u_{0}-u\right)^{2}+w_{1} \times\left(u_{1}-u\right)^{2}$;

Find the maximum variance of the corresponding gray level $t$ is the best image segmentation threshold.

The algorithm results are shown in Fig.10 and Fig.11. 


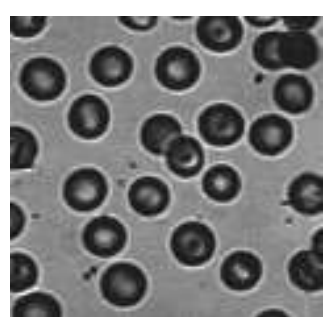

Figure 10. Binarization original map

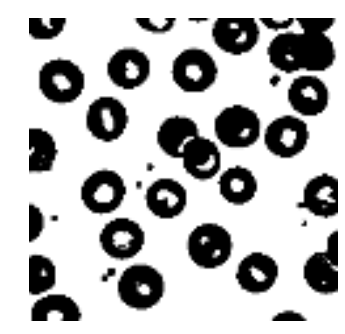

Figure 11. Binarization effect map

\section{Conclusion}

In this paper, the image processing platform based on TMS320C6713DSP is used to study the commonly used algorithm of image processing with 8-bit gray image as the research object, including image preprocessing, image segmentation and object image extraction. However, there are still many problems that need to be further studied in some identification processes and applications. Image segmentation is a difficult but very important problem, especially for complex background color image segmentation. Because the images obtained in the dynamic environment are affected in many ways, the challenge is accurate and effective. This paper examines the target detection in a static context. In practical applications, most of the target detection under dynamic background, there is a complex relative motion between the target and the $\mathrm{CCD}$, and the real-time updating of the background image must be considered.

\section{Acknowledgement}

This work was financially supported by the Henan Science and Technology Key Project (122102210563 and 132102210215).

\section{References}

[1] S.D.Mang, Z.Y.Zhang: Computer Vision - Theory and Algorithm Basis (Science Press, China 1997), p.52.

[2] Z.H.Liu, X.R.Wang, D.F. Wang. Computer age, (2015) No.9, p.21.

[3] Z.Z. Wu: Research on Image Signal Processing Technology Based on DSP (Ph.D., Northwestern Polytechnic University, China 2003), p.28.

[4] R.C.Zhao: Introduction to Digital Image Processing (Northwestern Polytechnic University Press, China 2000), p.83.

[5] Y.H.Jia: Computer Image Processing and Analysis (Wuhan University Press, China 2001), p.3.

[6] Y.J.Zhang: Image segmentation (Science Press, China 2001), p.23.

[7] S.Q.Han, L.Wang. Systems Engineering and Electronics, (2002) No.24, p.91.

[8] W.Wu, J.S.Wang. Journal of Xi' an University of Engineering Science and Technology, Vol. 19 (2002) No.1, p.86.

[9] K.X.Dai, G.H.Li. Journal of Image and Graphics, Vol. 11 (2006) No.7, p.919.

[10]Z.L.Fu. Computer Applications, Vol. 20 (2000) No.5, p.37.

[11]Q.S.Fan. Coal Technology, Vol. 32 (2013) No.9, p.254.

[12]D.L.Kong. Journal of Shandong Vocational College of Water Conservancy, (2012) No.4, p.11.

[13]B.Q.Chen. Journal of Jishou University (Natural Science Edition), (2009) No.1, p.63.

[14]G.J.Lv. Computer Knowledge and Technology, (2012) No.33, p.8035. 